\title{
Use of Shear Wave Elastography to Evaluate Stress Urinary Incontinence in Women
}

\author{
Nefise Tanrıdan $\mathrm{Okcu}^{1}$, Ediz Vuruskan² and Feride Fatma Gorgulu ${ }^{3}$ \\ ${ }^{1}$ Department of Obstetrics and Gynaecology, University of Health Sciences, Adana City Training and Research Hospital, Adana, Turkey \\ ${ }^{2}$ Department of Urology, University of Health Sciences, Adana City Training and Research Hospital, Adana, Turkey \\ ${ }^{3}$ Department of Radiology, University of Health Sciences, Adana City Training and Research Hospital, Adana, Turkey
}

\begin{abstract}
Objective: To compare the shear wave elastography (SWE) values of perineal tissues in female patients with stress urinary incontinence and those without incontinence.

Study Design: Prospective case control study.

Place and Duration of Study: University of Health Sciences, Adana City Training and Research Hospital, Adana, Turkey from March 2019 to March 2020.

Methodology: Seventy women with stress urinary incontinence ranging between 40-70 years; and 30 women of similar age and weight without complaints of incontinence were selected as cases and control group, respectively. SWE values of the external urethral sphincter, bladder neck, mid-urethral and pubococcygeal muscle regions were measured dynamically, both at rest and during Valsalva manoeuver by transperineal ultrasonography. Moreover, the medial pubic symphysis of the participants was taken as a fixed point and the angle between the bladder neck and urethra was measured at rest and during Valsalva. Patients with incontinence were divided into groups, mild and severe, according to the bladder stress test results.

Results: The angle change was statistically significantly higher in the severe and mild groups than the control group ( $p$ $<0.001$ ). There was no statistically significant difference between the bladder neck region elastography values in Valsalva manoeuver between the control group and the mild group, but the difference in the severe group was statistically significantly lower $(p=0.005$ ). No statistically significant difference was found between the control group and the mild group in terms of the mid-urethral region values at rest, but the difference in the severe group was statistically significantly lower ( $p<0.001$ ).

Conclusion: SWE is a promising new imaging method in the evaluation of urethral hypermobility in stress urinary incontinence.
\end{abstract}

Key Words: Ultrasonography, Shear wave elastography, Stress urinary incontinence, Transperineal ultrasonography.

How to cite this article: Okcu NT, Vuruskan E, Gorgulu FF. Use of Shear Wave Elastography to Evaluate Stress Urinary Incontinence in Women. J Coll Physicians Surg Pak 2021; 31(10):1196-1201.

\section{INTRODUCTION}

Urinary incontinence is a medical condition with social issues. Its prevalence has been reported to be approximately 5.9 $6.7 \%$. ${ }^{1}$ Continence occurs through the complex mechanism of normal anatomical and neurophysiological functions of the bladder, urethra and pelvic floor. ${ }^{2}$ Bladder and bladder neck support is mainly ensured by passive support of the anterior vaginal wall and active support of the levator ani muscles. Moreover, the levator ani is a striated muscle consisting of a thin layer of iliococcygeus in the lateral and the puborectal and pubococcygeus muscle groups in the medial. ${ }^{3}$

Correspondence to: Dr. Nefise Tanrldan Okcu, Department of Obstetrics and Gynaecology, University of Health Sciences, Adana City Training and Research Hospital, Adana, Turkey

E-mail: nefise-tanridan@hotmail.com

Received: May 04, 2021; Revised: August 17, 2021;

Accepted: September 17, 2021

DOI: https://doi.org/10.29271/jcpsp.2021.10.1196
Bladder neck closure occurs as a result of compression of the bladder neck to the pubovesical ligament in the normal retropubic position. It has been reported that deterioration and damage in the tissues that provide bladder neck support are associated with stress urinary incontinence (SUI). ${ }^{4}$ Additionally, it has been found that, in the periurethral connective tissues of women with SUI, collagen metabolism changes and collagen expression decrease significantly. ${ }^{5}$

The degree of anatomic displacement in the bladder neck has beenevaluated using a variety of methods, such as digital examination, the Q-tip test and transvaginal or transperineal ultrasonography. Among these methods, transperineal ultrasonography has been employed more frequently in recent years because it enables dynamic evaluation of tissues, it is easy to apply, it does not use radiation, and it does not cause application difficulties for patients. ${ }^{6,7}$

The evaluation of the pelvic floor with transperineal ultrasound can be done dynamically, so that in cases where intraabdominal pressure increases, anatomical displacement in the bladder neck can be easily monitored if the practitioner is experienced 
in imaging; and objective data can be provided for bladder displacement. ${ }^{8,9}$

Shear wave elastography (SWE) is a new imaging method and one of the types of ultrasound elastography that provides dynamic evaluation of tissue elasticity and stiffness. Although relatively more studies have been conducted on the differentiation of liver cirrhosis, malignancy in breast and thyroid masses and adenomyosis-leiomyoma in the uterus, only a limited number of studies have investigated its use in assessment of the pelvic floor. ${ }^{10-13}$

To the best of authors' knowledge, no previous study has investigated SWE in SUI. In the present study, it is aimed to compare the SWE values of perineal tissues in women with SUI and women without incontinence to investigate whether there is a difference between tissues and to determine if this method can aid in diagnosis.

\section{METHODOLOGY}

The study was planned as a prospective, case-control study. Ethics Committee approval (Date: 27/02/2019, Number: 29/399) for the study was obtained from the Research Hospital's Ethics Committee. Female patients, who were admitted to the Research Hospital's Gynaecology and Urology outpatient clinics between March 2019 and March 2020 due to urinary incontinence, were evaluated for the study. The patients were evaluated using a urinary incontinence, evaluation form, physical examination and bladder stress test. Patients between the ages of 40 - 70 years, who had a positive bladder stress test, had urinary incontinence with movements, such as cough and sneezing, for at least one year and had no urgency complaints, were included in the study. Thirty female patients of similar age and weight without complaints of incontinence, who agreed to be included in the study, were selected as the control group. All the women, who agreed to participate in the study, signed an informed consent form. Patients with chronic diseases, such as diabetes mellitus, heart failure, multiple sclerosis, using drugs that affect the bladder function, such as diuretic and anticholinergic usage, and those who previously had bladder surgery were excluded from the study.

The external urethral region, bladder neck, mid-urethral and pubococcygeal muscle region SWE values were measured dynamically both at rest and during Valsalva manoeuvers by transperineal ultrasonography in 70 female patients with SUI, and in 30 women without incontinence in the control group (Figure 1). The pubic symphysis was taken as a fixed point in mid-sagittal imaging, the angle between the bladder neck; and urethra was measured at rest and during Valsalva manoeuvers in each participants (Figure 1).

Transperineal ultrasonography was performed using the EPIQ 7 high-resolution ultrasonography device (Philips Healthcare, Inc., Andover, MA, USA) with a C5-1 16 MHz high-resolution convex probe in the puborectal-symphysis plane at rest and during the Valsalva manoeuvers (Figure 2). SWE was evaluated in the study subjects using the same transabdominal convex ultrasonog- raphy probe mentioned above with ElastPQ software based on acoustic radiation force impulse (ARFI) technology and employing the ElastPQ technique in the lithotomy position measured with the transperineal approach. ElastPQ is a point shear wave elastography (pSWE) technique implemented in the ultrasound systems of the Philips Healthcare. ElastPQ is an easy-to-use method of obtaining tissue stiffness values on a predefined region of interest (ROI). Using real-time imaging as a guide, the ROI is placed over the area of interest so the tissue stiffness data are obtained and displayed in seconds. Multiple samples can be recorded and targetted tissue report can be generated from the results. During ultrasonography imaging, the least possible pressure was applied with the probe. Measurement was ensured with the initial specification of a target (ROI) on a conventional ultrasound image. The ROI was placed and measured separately for each of the bladder neck, external urethral sphincter, pubococcygeus muscle region, and midurethral regions. In this SWE analysis, the continuous ROI box size was $13 \times 10 \mathrm{~mm}^{2}$. The results are stated as kilopascal ( $\left.\mathrm{kPa}\right)$. In addition, the angle between the bladder neck and urethra in the same position was measured by gray-scale ultrasonography.

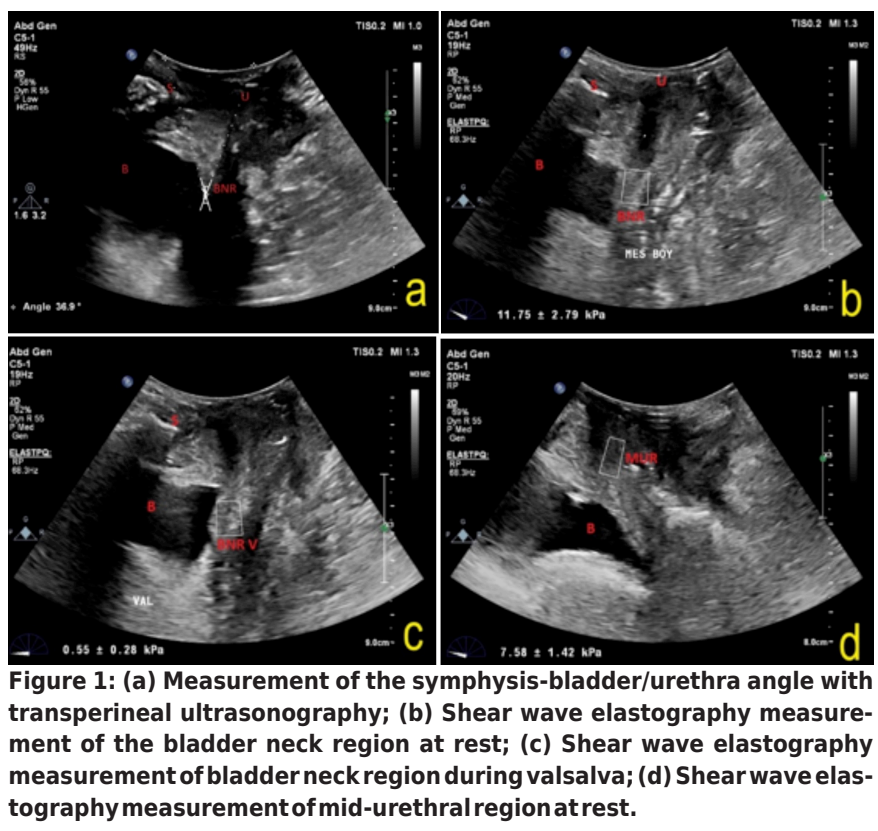

All the sonographic and elastographic evaluations were done by the same radiologist (FFG). The radiologist was blinded to the anamnesis and the physical examination results of the participants.

The patients with incontinence were divided into a mild incontinence group and a severe incontinence group, according to the bladder stress test result. The elastography and pubic symphysis-bladder neck/urethra angle values were compared between the mild incontinence, severe incontinence and control groups.

Data were given as mean \pm S.D or median (IQR: 25 th percentile-75th percentile). Normality control of the continuous variables was evaluated using the Shapiro-Wilk test. Levene's test was used to examine the homogeneity of the variances in the variables suitable for normal distribution. In the variables, where the 
variances were not homogeneously distributed, differences between the groups were determined using the Brown Forsythe test; Tamhane was used as a post hoc test. The one-way ANOVA test was used for the variables with homogeneous variances were homogeneous. The difference between the groups was compared with the Kruskal-Wallis test for the variables that did not conform to normal distribution. Pearson's correlation coefficient was calculated when examining the linear relationship between the two continuous variables; receiver operating characteristic (ROC) analysis was utilised to define the cut-off point for the bladder neck region elastography value ( $\mathrm{kPa}$ ) between the patient groups and the control group. P-values $<0.05$ were considered statistically significant. SPSS version 21 and MedCalc Software were used for the data analysis.

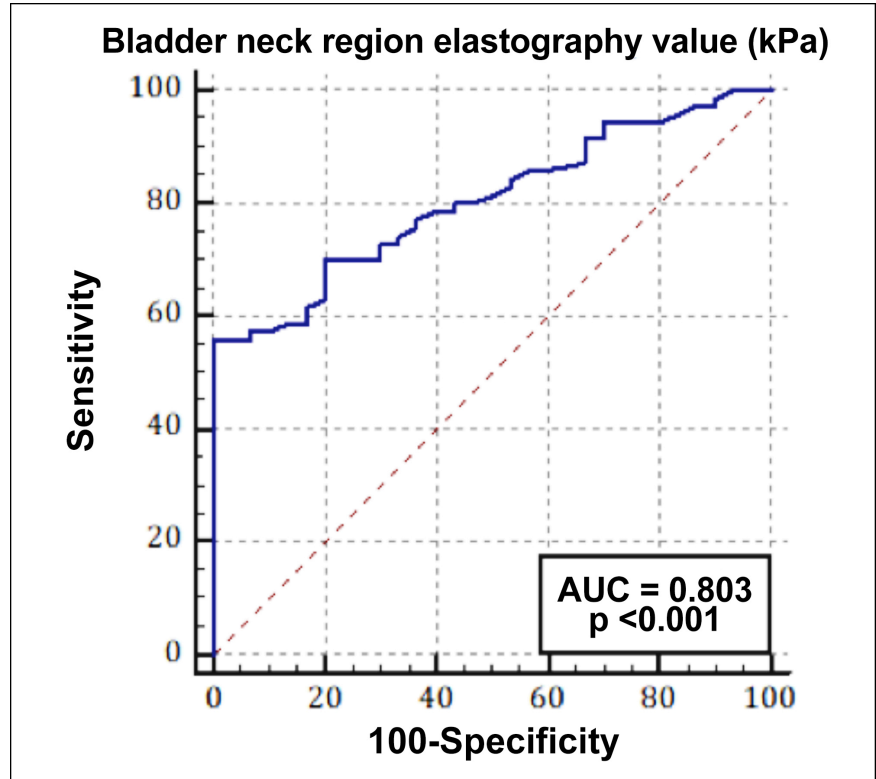

Figure 2: ROC analysis result for the bladder neck region elastography value in the stress urinary incontinence groups and the control group.

\section{RESULTS}

A total of 85 patients with SUI agreed to be included in the study. Fifteen patients were excluded due to diabetes mellitus, use of diuretics, or previous bladder surgery. The baseline characteristics of the patients are shown in Table I. No statistically significant difference was found between the two patient groups in terms of age in comparison to the control group, but the average age was higher in the severe incontinence group than the mild incontinence group ( $p=0.006$, Table I). Parity was higher in the severe and mild incontinence groups than the control group $(p<0.001$, Tablel).

The groups' mean \pm SD values of pubic symphysis-bladder neck/urethra (PS-BN/U) angle (rest), PS-BN/U angle (Valsalva), angle difference between rest and Valsalva, bladder neck region elastography (BNR-E in $\mathrm{kPa}$ at rest, BNR-E in $\mathrm{kPa}$ with Valsalva, externalurethral region elastography (EUR-E) atrestand withValsalva, mid-urethral region elastography (MUR-E) at rest and pubococcygeal muscle region elastography (PCR-E) at rest are shown in Tablell.
There was no significant difference between the severe and mild incontinence groups in terms of angle change, but the angle change was statistically significant in the severe and mild incontinence groups higher than the control group ( $p<0.001$, Table II). There was no statistically significant difference between the BNR-E values during Valsalva between the control and mild incontinence groups, but the difference in the severe incontinence group was statistically significantly lower ( $p=$ 0.005 , Table II). There was no statistically significant difference between the control group and the mild incontinence group in terms of the MUR-E values at rest, but the difference in the severe incontinence group was statistically significantly lower ( $p<0.001$, Table II).

Moreover, when comparing the incontinent groups with the non-incontinent group, the correlation between BNR-E, MUR-E with PS-BN/U angle and angle difference was examined at rest and during Valsalva. A statistically significant correlation was found between the PS-BN/U angle with BNR-E (rest and Valsalva) and MUR-E (rest) values in the incontinent patient group ( $p<0.001$, Table III). It was observed that, as the angle and angle difference increased, the elastography values decreased in these regions (Table III).

ROC analysis was performed for the use of SWE in the evaluation of bladder neck hypermobility in SUI. The cut-off was determined as $\leq 7.04 \mathrm{kPa}$ in the differentiation of SUI from the control group. According to this value, the sensitivity was $55.7 \%$ (95\% confidence interval [CI]: [43.3-67.6]), specificity was $100 \%$ (95\% Cl: [88.4-100.0]); AUC =0.803 [95\% Cl: 0.71-0.87], p $<0.001$, Figure 3).

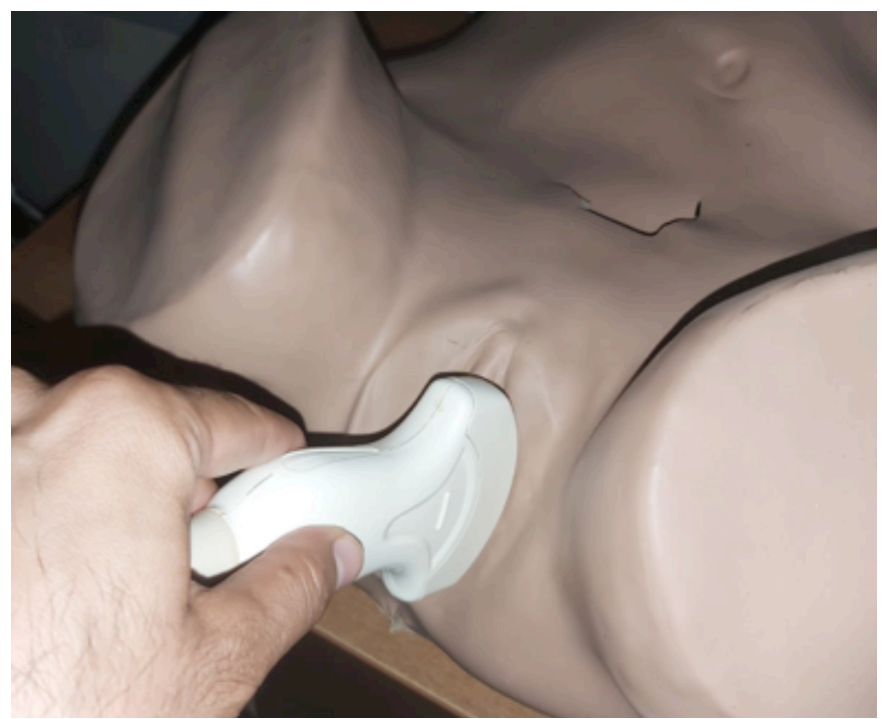

Figure 3: Transducer placement fortransperineal ultrasonography.

\section{DISCUSSION}

This study results demonstrated that the use of SWE in SUI can be a helpful contribution to the diagnosis. To the best of authors' knowledge, this is the first study in which the urethral, periurethral and coccygeal muscle regions were evaluated with SWE in SUI, and compared with the control group. 
Table I: Baseline characteristics of the three groups.

\begin{tabular}{|c|c|c|c|c|}
\hline & Control & Mild & Severe & \multirow[b]{2}{*}{$\mathbf{p}$} \\
\hline & $\begin{array}{c}\text { Mean } \pm \text { SD } \\
\text { Median [IQR] }\end{array}$ & $\begin{array}{c}\text { Mean } \pm \text { SD } \\
\text { Median [IQR] }\end{array}$ & $\begin{array}{c}\text { Mean } \pm \text { SD } \\
\text { Median [IQR] }\end{array}$ & \\
\hline Age & $61 \pm 8.87[65]$ & $57.63 \pm 8.4[58]$ & $64.18 \pm 5.32[65]^{b}$ & $0.006^{*}$ \\
\hline $\mathrm{BMI}$ & $28.59 \pm 4.06$ & $29.03 \pm 4.06$ & $27.9 \pm 3.06$ & 0.528 \\
\hline Parity & $2.5 \pm 1.04 . \quad 2.5[2-3]$ & $3.4 \pm 1.09[3]^{a}$ & $4.45 \pm 1.87[5]^{a}$ & $<0.001^{*}$ \\
\hline
\end{tabular}

Table II: Angle and elastography values at rest and during Valsalva among the three groups.

\begin{tabular}{|c|c|c|c|c|}
\hline & $\begin{array}{l}\text { Control } \\
(n=30)\end{array}$ & $\begin{array}{c}\text { Mild } \\
(n=48)\end{array}$ & $\begin{array}{l}\text { Severe } \\
(n=22)\end{array}$ & \multirow[t]{2}{*}{$\mathbf{p}$} \\
\hline & Mean \pm SD & Mean \pm SD & Mean \pm SD & \\
\hline PS-BN/U angle (degree, ${ }^{\circ}$ ) (rest) & $45.27 \pm 4.18$ & $58.71 \pm 7.21^{a}$ & $68.05 \pm 6.18^{a b}$ & $<0.001$ \\
\hline PS-BN/U angle (degree, ${ }^{\circ}$ ) (valsalva) & $55.47 \pm 5.39$ & $79.19 \pm 10.01^{a}$ & $87.91 \pm 8.11^{a b}$ & $<0.001$ \\
\hline AD (valsalva-rest) (degree, $\left.{ }^{\circ}\right)$ & $10.23 \pm 2.33$ & $20.56 \pm 7.19^{a}$ & $19.91 \pm 5.69^{a}$ & $<0.001$ \\
\hline BNR-E value (kPa) (rest) & $11.96 \pm 3.42$ & $8.82 \pm 3.95^{a}$ & $2.54 \pm 0.95^{a b}$ & $<0.001$ \\
\hline BNR-E value (kPa) (valsalva) & $3.36 \pm 2.24$ & $2.90 \pm 1.41$ & $1.81 \pm 1.12^{\mathrm{ab}}$ & 0.005 \\
\hline EUR-E value (kPa) (rest) & $11.41 \pm 2.74$ & $8.87 \pm 3.67^{a}$ & $4.65 \pm 1.86^{a b}$ & $<0.001$ \\
\hline EUR-E value (kPa) (valsalva) & $10.1 \pm 2.76$ & $8.13 \pm 3.53^{a}$ & $4.32 \pm 2^{a b}$ & $<0.001$ \\
\hline MUR-E value (kPa) (rest) & $12.47 \pm 4.53$ & $12.05 \pm 4.39$ & $6.27 \pm 2.17^{\mathrm{ab}}$ & $<0.001$ \\
\hline PCMR-E value (kPa) (rest) & $10.06 \pm 2.88$ & $10.58 \pm 3.86$ & $6.49 \pm 1.96^{\mathrm{ab}}$ & $<0.001$ \\
\hline
\end{tabular}

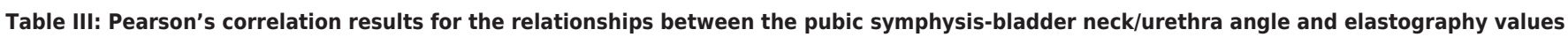
in the stress urinary incontinence groups and the control group.

\begin{tabular}{|c|c|c|c|c|c|c|}
\hline \multirow{2}{*}{\multicolumn{2}{|c|}{ Patients }} & \multicolumn{5}{|c|}{ Control } \\
\hline & & \multirow[t]{2}{*}{$\begin{array}{c}\text { PS-BN/U } \\
\text { Angle (V) }\end{array}$} & \multirow{2}{*}{$\begin{array}{c}\text { AD } \\
\text { (V-R) }\end{array}$} & \multirow{2}{*}{$\begin{array}{c}\begin{array}{c}\text { BNR-E } \\
\text { value (R) }\end{array} \\
-0.520\end{array}$} & \multirow{2}{*}{$\begin{array}{c}\text { MUR-E } \\
\text { value (R) } \\
-0.364\end{array}$} & \multirow{2}{*}{$\begin{array}{c}\begin{array}{c}\text { BNR-E } \\
\text { value (V) }\end{array} \\
-0.343\end{array}$} \\
\hline PS_RN/II angle (Y) & $r$ & & & & & \\
\hline PS-BIV/U angie (V) & $p$ & & $<0.001$ & 0.003 & 0.048 & 0.063 \\
\hline \multirow{2}{*}{$A D(V-R)$} & $r$ & 0.606 & & -0.761 & -0.742 & -0.467 \\
\hline & $p$ & $<0.001$ & & $<0.001$ & $<0.001$ & 0.009 \\
\hline \multirow{2}{*}{ BNR-E value $(R)$} & $r$ & -0.536 & -0.768 & & 0.473 & 0.489 \\
\hline & $\mathrm{p}$ & $<0.001$ & $<0.001$ & & 0.008 & 0.006 \\
\hline \multirow{2}{*}{ BNR-E value (V) } & $r$ & -0.559 & -0.793 & 0.941 & 0.718 & \\
\hline & $\mathrm{p}$ & $<0.001$ & $<0.001$ & $<0.001$ & $<0.001$ & \\
\hline \multirow{2}{*}{ MUR-E value (R) } & $r$ & -0.537 & -0.578 & 0.779 & & 0.261 \\
\hline & $p$ & $<0.001$ & $<0.001$ & $<0.001$ & & 0.164 \\
\hline
\end{tabular}

Al-Saadi et al. evaluated the angle between the proximal urethra and pubic symphysis of patients with SUI. They found that this angle increased in patients with urinary incontinence. $^{8}$

The findings of this study are consistent with those results. There was a correlation between the angle difference with the resting BNR-E and MUR-E values $(\mathrm{kPa})$. It was also found that as the angle difference increased, the elastography values decreased.

When elastography is performed, objective data can be obtained in addition to ultrasonographic anatomical evaluation regarding tissue elasticity and stiffness. Elastography research on pelvic floor diseases is extremely scarce. A limited number of studies have evaluated pelvic floor struc- 
tures, such as levator ani, urethral sphincter or anal sphincter muscles; and it has been stated that more studies are needed on this subject. ${ }^{9,14}$

Kreutzkamp et al. evaluated patients with incontinence using strain elastography; they reported that the elasticity of the tissues of patients differed, and as the elastography rates decreased, the urethral mobility increased. ${ }^{15}$ This study results are also consistent with those findings. However, in that study, the patients were not separated according to their incontinence types and the patients with signs of urge incontinence were also included in that study. This factor may have affected the study results for SUI, where tissue elasticity may be much more important. Furthermore, this condition was specified as a limitation for their study, so we tried to prevent this limitation by distinguishing the type of incontinence as urge and stress at the beginning of this study. In addition, in this study, the SWE ( $\mathrm{kPa}$ ) values of the mild incontinence and severe incontinence groups and the control group were compared. A statistically significant difference was found between all three groups in terms of the SWE ( $\mathrm{kPa}$ ) values of the bladder neck region ( $p<0.001$ ). Moreover, the SWE cutoff value of the bladder neck region was found $\leq 7.04 \mathrm{kPa}$ in the diagnosis of SUI and at this value SUI detection sensitivity was $55 \%$, specificity was $100 \%$ (AUC $=0.803, p<0.001$ ).

In this study, SWE was used, which is a dynamic method, without applying pressure. There was only one study in the literature that had evaluated female urogenital sphincter with SWE. That pilot study was conducted on 10 women without incontinence, using SWE technique in the urogenital sphincter. $^{16}$

Pavlov et al. reported that the amount of type 4 collagen in the vaginal and perineal tissues of patients with SUI is significantly reduced and it may affect physiopathology. ${ }^{17}$

In this study, data was obtained based on bladder displacement and the elasticity and stiffness of periurethral tissues in a dynamic, non-invasive manner without pathological evaluation and without applying pressure using SWE.

A study comparing the pelvic trauma index after vaginal delivery and Caesarean delivery with strain elastography reported that the trauma index was higher after vaginal delivery. ${ }^{18}$ In this study, one of the baseline characteristics, and the number of parities, were statistically significantly higher in patients with severe incontinence. In this respect, the statistically lower SWE ( $\mathrm{kPa})$ values on the bladder neck, mid-urethral and pubococcygeal muscle regions $(p<0.001)$ in the present study are consistent with the results of that study. ${ }^{18}$

Given that the muscle boundaries are not separated sharply at rest and during Valsalva, it is difficult to assess specific areas of the pelvic floor. This situation becomes more pronounced during Valsalva, and it may be considered to be a limitation of this study. Another limitation is that there may be changes in the values at different Valsalva pressures. However, since SWE gives more quantitative results, repeatability is the advantage of this method.

\section{CONCLUSION}

SWE is a promising new imaging modality that can provide rapid diagnosis in the evaluation of urethral hypermobility, which associated with anatomical stress urinary incontinence pathophysiology. SWE can also be used to identify patients who need treatment for SUI and follow-up examinations. Studies with larger patient groups are needed to achieve standardisation and to establish cut-off values in the diagnosis of SUI.

\section{ETHICAL APPROVAL:}

Ethics Committee approval was obtained before starting the study from the Clinical Research Ethics Committee of the University of Health Sciences, Adana City Training and Research Hospital on 27/02/2019 with decision number 29/399.

\section{PATIENTS' CONSENT:}

Informed consent forms were obtained from all women who agreed to participate in the study.

\section{CONFLICT OF INTEREST:}

The authors declared no conflict of interest.

\section{AUTHORS' CONTRIBUTION:}

NTO, FFG: Concept, design.

FFG: Supervision.

NTO, EV: Data collection, processing and interpretation and critical reviews.

NTO, EV, FFG: Literature search and writing manuscript.

\section{REFERENCES}

1. Komesu YM, Schrader RM, Ketai LH, Rogers RG, Dunivan GC. Epidemiology of mixed, stress, and urgency urinary incontinence in middle-aged/older women: The importance of incontinence history. Int Urogynecol J 2016; 27(5): 763-72. doi: 10.1007/s00192-015-2888-1.

2. Ptaszkowski K, Paprocka-Borowicz M, Słupska L, Bartnicki J, Dymarek R, Rosińczuk J, et al. Assessment of bioelectrical activity of synergistic muscles during pelvic floor muscles activation in postmenopausal women with and without stress urinary incontinence: A preliminary observational study. Clin Interv Aging 23; 10:1521-8. doi: 10.2147/CIA. S89852.

3. Gowda SN, Bordoni B. Anatomy, abdomen and pelvis, levator ani muscle. [Updated 2021 Feb 7]. In: StatPearls [Internet]. Treasure Island (FL): StatPearls Publishing; 2021 Jan-. Available from: http://www.ncbi.nlm.nih.gov/books/ NBK556078/.

4. Falah-Hassani K, Reeves J, Shiri R, Hickling D, McLean L. The pathophysiology of stress urinary incontinence: A systematic review and meta-analysis. Int Urogynecol J 2021; 32(3):501-52. doi: 10.1007/s00192-020-04622-9.

5. Goepel C, Hefler L, Methfessel HD, Koelbl H. Periurethral 
connective tissue status of postmenopausal women with genital prolapse with and without stress incontinence. Acta Obstet Gynecol Scand 2003; 82(7):659-64. doi: 10.1034/j. 1600-0412.2003.00019.x.

6. Yang X, Zhu L, Li W, Sun X, Huang Q, Tong B, et al. Comparisons of electromyography and digital palpation measurement of pelvic floor muscle strength in postpartum women with stress urinary incontinence and asymptomatic parturients: A cross-sectional study. Gynecol Obstet Invest 2019; 84(6):599-605. doi: 10.1159/000501825.

7. Sendag F, Vidinli H, Kazandi M, Itil IM, Askar N, Vidinli B, et al. Role of perineal sonography in the evaluation of patients with stress urinary incontinence. Aust N Z J Obstet Gynaecol 2003; 43(1):54-7. doi: 10.1046/j.0004-8666. 2003.00012.x.

8. Al-Saadi WI. Transperineal ultrasonography in stress urinary incontinence: The significance of urethral rotation angles. Arab J Urol 2016; 14(1):66-71. doi: 10.1016/j. aju.2015. 11.003.

9. Jamard E, Blouet $M$, Thubert $T$, Rejano-Campo M, Fauvet $R$, Pizzoferrato AC. Utility of 2D-ultrasound in pelvic floor muscle contraction and bladder neck mobility assessment in women with urinary incontinence. J Gynecol Obstet Hum Reprod 2020; 49(1):101629. doi: 10.1016/j.jogoh.2019. 101629.

10. Akyuz M, Gurcan Kaya N, Esendagli G, Dalgic B, Ozhan Oktar S. The evaluation of the use of 2D shear-wave ultrasound elastography in differentiation of clinically insignificant and significant liver fibrosis in pediatric age group. Abdom Radiol (NY) 2021; 46(5):1941-6. doi: 10.1007/ s00261-020-02844-5.

11. Gürüf A, Öztürk M, Bayrak IK, Polat AV. Shear wave versus strain elastography in the differentiation of benign and malignant breast lesions. Turk J Med Sci 2019; 49(5):
1509-1517. doi: 10.3906/sag-1905-15.

12. Zhao CK, Chen SG, Alizad A, He YP, Wang Q, Wang D, et al. Three-dimensional shear wave elastography for differentiating benign from malignant thyroid nodules. J Ultrasound Med 2018; 37(7):1777-88. doi: 10.1002/ jum.14531.

13. Görgülü FF, Okçu NT. Which imaging method is better for the differentiation of adenomyosis and uterine fibroids? J Gynecol Obstet Hum Reprod 2020; 50(5):102002. doi: 10.1016/j.jogoh.2020.102002.

14. Ozer N, Gorgulu FF. Evaluation of the mechanical properties of anorectal tissues and muscles by shear wave elastography in anal fissure disease. J Coll Physicians Surg Pak 2020; 30(11):1133-7. doi: 10.29271/jcpsp.2020. 11.1133.

15. Kreutzkamp JM, Schäfer SD, Amler S, Strube F, Kiesel L, Schmitz R. Strain elastography as a new method for assessing pelvic floor biomechanics. Ultrasound Med Biol 2017 Apr; 43(4):868-72. doi: 10.1016/j.ultrasmedbio. 2016.12.004.

16. Aljuraifani R, Stafford RE, Hug F, Hodges PW. Female striated urogenital sphincter contraction measured by shear wave elastography during pelvic floor muscle activation: Proof of concept and validation. Neurourol Urodyn 2018; 37(1):206-212. doi: 10.1002/nau.23275.

17. Pavlov VN, Yashchuk AG, Kazikhinurov AA, Musin II, Zauinullina RM, Kulavskii VA, et al. Structural-morphological changes of the connective tissue of the vaginal mucosa and perineal skin in women with stress urinary incontinence. Urologiia 2017; 5:15-20. doi: 10.18565/ urology.2017.5. 15-20.

18. Maßlo K, Möllers M, de Murcia KO, Klockenbusch W, Schmitz R. New method for assessment of levator avulsion ınjury: A comparative elastography study. J Ultrasound Med 2019; 38(5):1301-1307. doi: 10.1002/jum.14810. 